\title{
Advanced enhancement model of bionics fish cilia MEMS vector hydrophone-systematic analysis review
}

\begin{abstract}
A bionic fish cilia median-low frequency three-dimensional MEMS vector hydrophone is reported in this paper. The piezo resistive reasonable position was obtained through finite element analysis by ANSYS and the structure was formed by MEMS processes including lithography, ion implantation, PECVD and etching etc. The standing wave barrel results show that the lowest sensitivity of the hydrophone is $-200 \mathrm{~dB}$ and reach up to $-160 \mathrm{~dB}$ (in which the voltage amplification factor is 300 ). It has a good frequency response characteristics in $25 \mathrm{~Hz} \square 1500 \mathrm{~Hz}$ band. Directivity tests displayed that the hydrophone has a good "8"-shaped directivity, in which the resolution is not less than $30 \mathrm{~dB}$, and asymmetry of the maximum axial sensitivity value is less than $1.2 \mathrm{~dB}$ and this hydrophone has a good directional pattern in form of "8"-shapes. The experiment results show that the receiving sensitivity of the hydrophone is $197.7 \mathrm{~dB}(0 \mathrm{~dB} \quad 1 / 4$ $1 \mathrm{~V} / \mathrm{mPa}$ ). The novel hydrophone not only possesses satisfactory directional pattern as well as miniature structure, but also has good low-frequency characteristics, and satisfies the requirements for low-frequency acoustic measurement.
\end{abstract}

Keywords: bionics, hydrophone, MEMS, acoustic measurement, biology
Volume 2 Issue 2 - 2018

\author{
Priyanka EB, Thangavel S \\ Department of Mechatronics Engineering, Kongu Engineering \\ College, India
}

Correspondence: Priyanka EB, Department of Mechatronics Engineering, Kongu Engineering College, Erode, India, Email priyankabhaskaran1993@gmail.com

Received: August 16,2017| Published: March 26, 2018

\section{Introduction}

Engineers, designers and architects often look to nature for inspiration. Biology has perfected its designs and formed many fruitful abilities such as its exquisite sensitivity, effectiveness, and reliability, through billions of years of evolution. So mimicking its creations is a sure way of producing new technologies and new achievement that are both efficient and reliable which leads to development of a threedimensional MEMS vector hydrophone based on fish cilia and piezo resistive principle. But the vector hydrophone with low-frequency detection, miniaturization, and high sensitivity is rarely reported in open published literatures. A kind of MEMS vector hydrophone is fabricated by our research group. Before improving, the receiving sensitivity of the hydrophone was $197.7 \mathrm{~dB}(0 \mathrm{~dB} 1 / 41 \mathrm{~V} / \mathrm{mPa})$, the hydrophone possesses directional pattern in the form of " 8 " shape and the resolution of the directivity pattern $\mathrm{Kd} 420 \mathrm{~dB}$. The articles published previously focused on the processing of MEMS vector hydrophone. ${ }^{1}$ At that time the overall performance of hydrophone was not ideal, which mainly embodied in lower sensitivity and greater fluctuant frequency response curve. Therefore this paper proposed four aspects to improve the performance of hydrophone. Firstly, fiber should be chosen as the cilium of the material to replace the previous plastic rod; secondly, mature diffusion technology of unit should be selected to ensure the consistency of resistance; thirdly, the density of polychloroprene should match with that of water; fourthly, a novel package type was proposed. Through the above improvements, the sensitivity has been increased by $12 \mathrm{~dB}$ and frequency response curve has also been greatly improved.

The vector hydrophone has azimuth elevation directivity independent of signal frequency and signal bandwidth, now vector hydrophone has been applied in the most passive detection. Leslie et al. fabricated a hydrophone measuring the water flow velocity by mounting a velocity pickup in a rigid, spherical housing, and it has quite directivity in low frequencies. ${ }^{2}$ Recently rapid progresses have been made in the particle velocity hydrophone. ${ }^{3}$ Fan et al. from the University of Illinois at Urbana-Champaign report the development of micro machined, distributed flow sensors based on the fish lateral line sensors. ${ }^{4}$ Izadi et al. ${ }^{5}$ in Universitat Bonn report the fabrication of aquatic hair based flow sensors inspired by fish lateral line. ${ }^{5}$ Ships et al. designed a 3-dimensional micro vector hydrophone for American navy. ${ }^{6,7}$ Boston University designed a MEMS hydrophone, which can detect the sound field variation by detecting the reflected laser beam. ${ }^{8}$ Ultrasonic hydrophone was also researched in recent years. Chan et al. ${ }^{9}$ fabricated a nanocomposite ultrasonic hydrophone, which has a high sensitivity at the frequency ranging from 1 to $10 \mathrm{MHz} .{ }^{9}$ Heerfordt et al., ${ }^{10}$ Bagnoli et al., ${ }^{11}$ developed the fiber hydrophone, which can work without electric pores.

The advantage of piezo resistive effect is that it can be used to detect low-frequency signal even at $0 \mathrm{~Hz}$. Therefore, it is of great advantage for research on vector hydrophone based on piezo resistive effect. In this paper, a novel MEMS vector hydrophone based on the theory of bionics and piezo resistive effect will be presented, with respect to the design, fabrication, and preliminary characterization. The targeted application region for these sensors is low-frequency detection of submarine sound.

\section{Sensor improvement}

Both the acoustic-electric transducer infrastructure and the acoustic package of hydrophone have been optimized, which has great effect on the performance of the novel MEMS vector hydrophone. The improvement work can be divided into two parts and described as the following.

A. The sensitivity and frequency of the hydrophone affected by the material of the cilia.

B. Among the acoustic-electric transducer infrastructure, the cilia play the role of perceiving the sound wave. That is, the cilia can 
directly sense the vibration of acoustic particles, then the vibration can be passed to the four-beam structure, and then the four-beam structure is led to deform. Therefore, the geometry of cilia also has certain requirements.

C. Material selection of the cilia according to the results derived from the co-vibration model, the density of the cilia should match the surrounding material (water here) in order to make the cilia vibrate with the acoustic particles Then considering the cilia take a role in transmitting the vibration of acoustic particle, so the glass optical fiber with greater stiffness is selected to replace the previous plastic cylinder with less stiffness. The fiber's density is $2.1 \mathrm{~g} / \mathrm{cm} 3$ and diameter is $150 \mu \mathrm{m}$.
D. The geometry determination of the cilia. Reference to one end fixed cantilever, ${ }^{8}$ it's known that first-order resonance frequency relates with the length of cantilever beam, the radius of beam, the density of material and Young's modulus.

\section{Analysis structure}

According to the bionic principle of a fish's lateral line, previous research has developed one kind of bionic package of MEMS vector hydrophone, ${ }^{9}$ as shown in Figure 1. When the sound signal is sensed by the cylinder, the piezo resistors located at the beam transform the sound signal into strain and finally into a differential output voltage signal via the Wheatstone bridge circuit. Thus, the sound signal can be detected. ${ }^{10}$

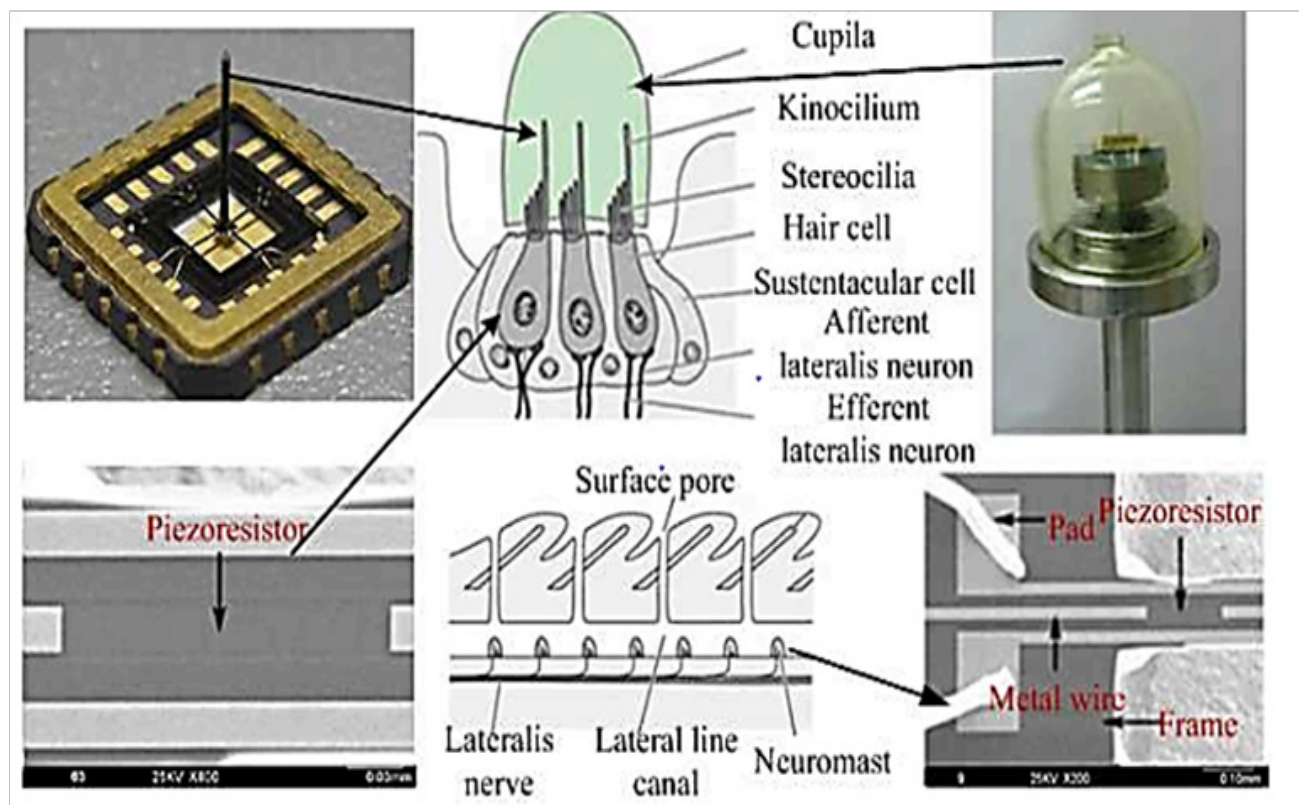

Figure I Bionic package of MEMS vector hydrophone.

\section{Design structure}

According to the auditory principle of lateral line organ, we can see that the mechanoreceptor structure is the stereocilia which acts as sensors for flow noise by stimulating the hair cell. Therefore, the bionic structure mainly includes the design of hair cell and its stereocilia. In this paper, the piezoresistor is simulated to hair cell and the rigid plastic cylinder is simulated to stereocilia. The structure of hydrophone consists of two parts: fourbeam microstructure and rigid plastic cylinder which has the same density as that of water. Figure 2 shows the actual design of this structure. Figure $2 \mathrm{~A}$ is the three-dimensional (3D) model of the design and Figure 2B gives the two dimensional (2D) top view of the design. The four-beam microstructure consists of four vertical cantilever beams. The rigid plastic cylinder is fixed at the center block of the four-beam microstructure. Both the center block and the beams have the same thickness. The whole structure has complete axial symmetry in the xoz plane and yoz planes. According to acoustics theory, only when the cylinder and the surrounding medium have the same density can the cylinder and the medium particle have synchronous vibration, or else the acoustic information cannot be exactly memorized. In this paper, the rigid plastic cylinder not only has the same density as that of water but also has small geometric size (diameter: $200 \mathrm{~mm}$, length: $5000 \mathrm{~mm}$ ), meeting the vibration conditions well.

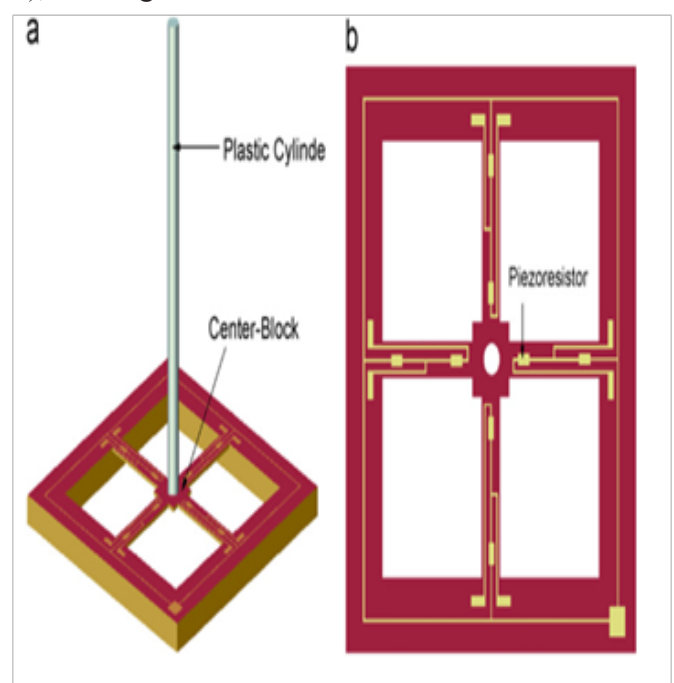

Figure 2 (A) 3D model and (B) 2D top view of the hydrophone structure. 


\section{Fabrication}

The fabrication of the hydrophone consists of the processing of four-beam microstructure and the manufacture of rigid plastic cylinder. The rigid plastic cylinder can be easily achieved by plastic molding processing. The four beam microstructure is manufactured by means of the silicon-on-insulator (SOI) wafer with MEMS technology. To define the metal tracks, the windows of the metal to silicon contacts have been opened using the same process as the implantation windows to reveal the underlying $\mathrm{p}$ type doped silicon. After the resist has been stripped in a fuming nitric acid solution, an aluminum-1\% silicon metal layer has been deposited on the wafers.

The metal layer has been patterned by photolithography and etched to define the metal tracks. The cantilevers in the device layer have been etched in an inductively coupled plasma (ICP) reactor after the $\mathrm{SiO}_{2}$ passivation layer has been removed and the $2 \mathrm{~mm}$ thick buried oxide has been subsequently removed. In the last step, the wafer has been aligned on the backside and the structures have been released by ICP process, and then the buried oxide layer has been removed by a dry etch step. The SEM image (top view) of the microstructure is shown in Figure 3. After the four-beam microstructure is fabricated, a rigid plastic cylinder is fixed at the center of the microstructure as shown in Figure 4, and then packaged in a hat which is made of soundtransparent polychloroprene rubber. A built-in high-quality, low noise, $50 \mathrm{~dB}$ preamplifier provides signal conditioning for transmission over long underwater cables. An integrated waterproof rubber ring allows quick disconnection of the cable and makes replacements and storage very easy. In order to make the motion of the rigid plastic cylinder be the same as that of the acoustic medium particle, castor oil that has the same density as that of water is poured into the hat and low-noise cable is led out.

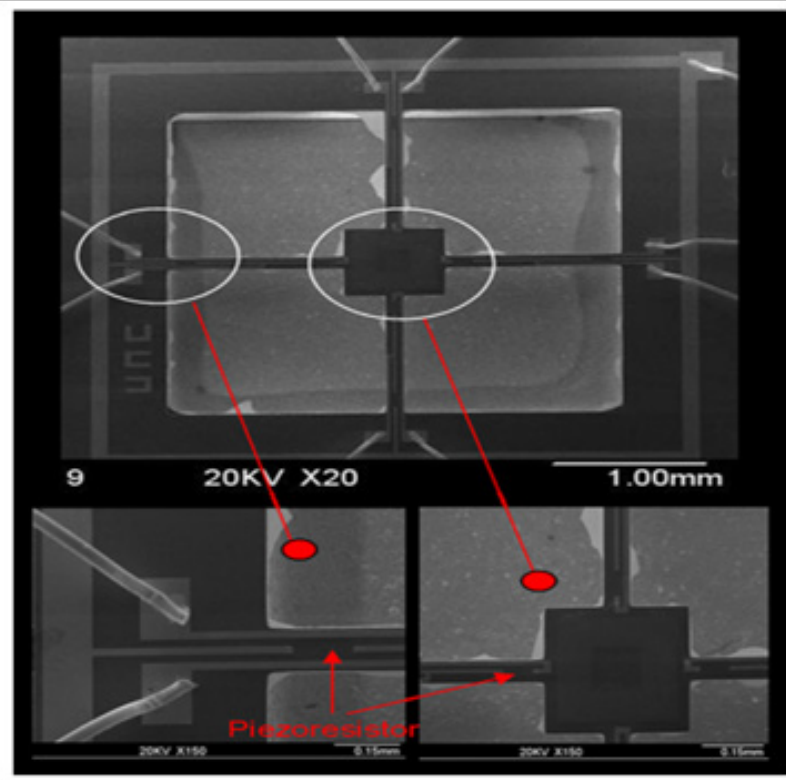

Figure 3 SEM images (top view) of microstructure.

\section{Simulation results}

It is well known, the piezo resistive effect represents the change in electrical resistivity of a semiconductor when mechanical stress is applied. The piezo resistance expression of silicon cantilever is

$$
\Delta \mathbf{R} / \mathbf{R}=\sigma \mathbf{l} \boldsymbol{l} \mathbf{l}+\sigma \mathbf{t} \pi \mathbf{t} .
$$

For the piezo resistors with P -type 110 crystal orientation,

$$
\pi \mathrm{l}=71.8 \times 10-11 \mathrm{~Pa}-1 \text {, and } \pi \mathrm{t}=66.3 \times 10-11 \mathrm{~Pa}-1 \text {, }
$$
respectively.

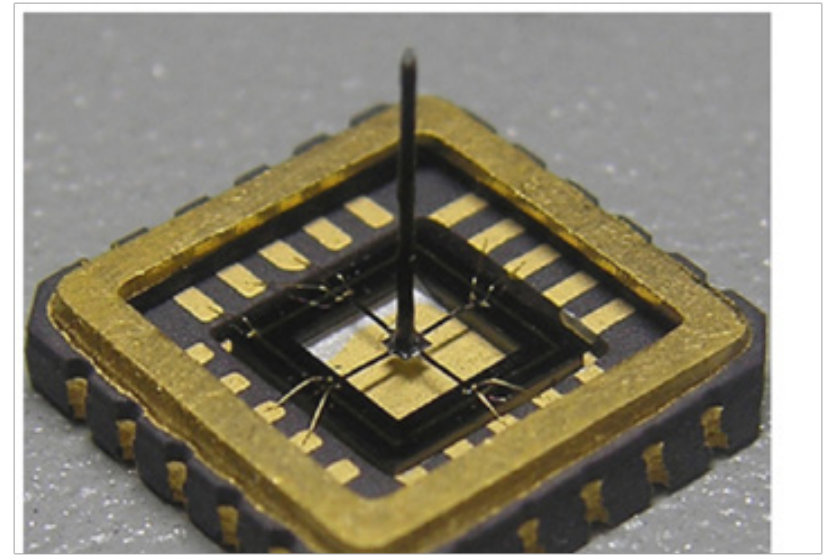

Figure 4 Photo of the hydrophone structure.

Generally, when external force acts on the cantilever, its shear stress $\sigma \mathrm{t}$ can be neglected for far less than normal stress $\sigma l$. So the mechanical sensitivity of the vector hydrophone would be expressed as: $\mathrm{S}=71.8 \times 10-11 \sigma \mathrm{lVin} / \mathrm{P}$ (where Vin is the input voltage, and $\mathrm{P}$ is the sound pressure). ${ }^{7}$ In order to obtain a maximum sensitivity, the piezo resistor cannot be placed in the maximum stress area and the nonlinear area. The stress of the MEMS hydrophone was analyzed using ANSYS where 1 Pa loads were added along the $\mathrm{Y}$ direction of the rigid cylinder and $\mathrm{Z}$ direction to the cantilever beam. The stress-contour of the structures are shown in Figures 5A \& Figure 6A, whereas, the stress curves of one beam obtained by path definition in ANSYS are shown in Figures 5B \& Figure $6 \mathrm{~B}$. According to the piezo resistor distribution principle, the simulation piezo resistor distribution graph was marked in Figures 5B $\&$ Figure 6B, where there were about $100 \mu \mathrm{m}$ from the end of crossbeam and $200 \mu \mathrm{m}$ from the roots of silicon cantilever. Moreover, the transverse stress of cross-beam $\mathrm{X}$ direction was approximated to zero in symmetrical distribution as shown in Figures 5 \& Figure 6.

\section{Acoustic analysis}

Figure 7 illustrates the cohesive energy model of "umbrella-type" packaged structure by ANSYS. It can be seen that the particle velocity is obviously much larger at the location closer to the optical fiber cylinder than that of the locations away from the optical fiber cylinder, which is agreement with the theoretical analysis. We extract the acoustic pressure at point A (inside the cap) with pre-packaged structure and "umbrella-type" packaged structure respectively in LMS virtual Lab acoustic. The sensitivity test adopts a standing wave comparison calibration, i.e., the open-circuit voltage output of the tested MEMS hydrophone is compared with the reference hydrophone to determine the sensitivity of the tested hydrophone. Figure 8 depicts the frequency response of the pre-packaged hydrophone and "umbrella"-type packaged hydrophone. It can be seen that the receiving sensitivity of the "umbrella"-type packaged hydrophone reaches - 178dB (0 
$\mathrm{dB}$ reference $1 \mathrm{~V} / \mu \mathrm{Pa}$ ), increased by $20 \mathrm{~dB}$ comparing with the prepackaged hydrophone, and that the frequency band ranges from $20 \mathrm{~Hz}$ to $2 \mathrm{KHz}$ (broaden one times).More importantly, the fluctuation of the curve is within $\pm 2 \mathrm{~dB}$ (decreased by $\pm 3 \mathrm{~dB}$ ).
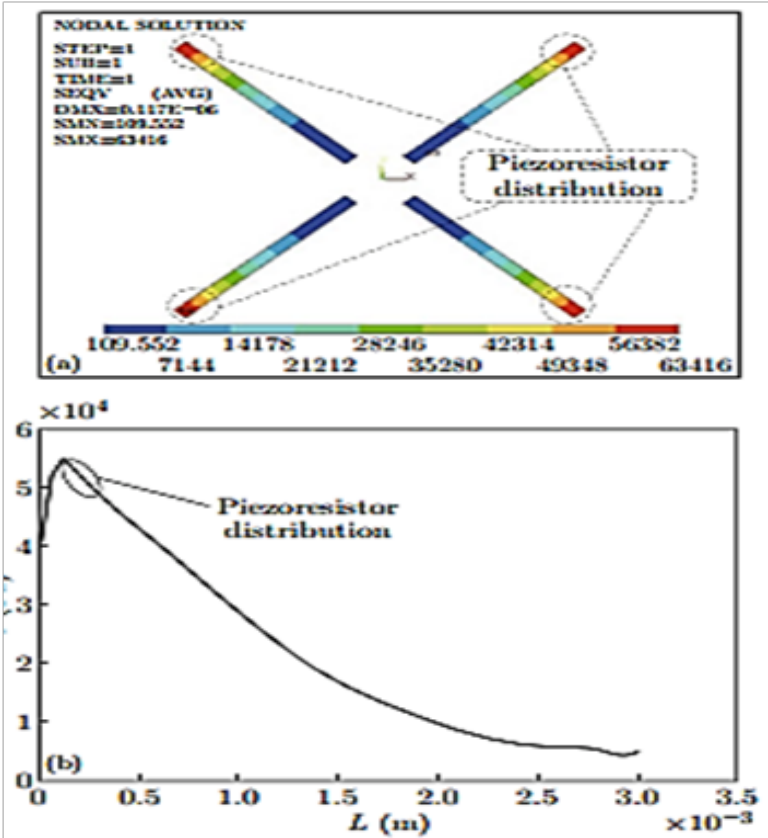

Figure 5 (A) The stress-contour of cantilever beam; (B) Curve of cantilever stress distribution.
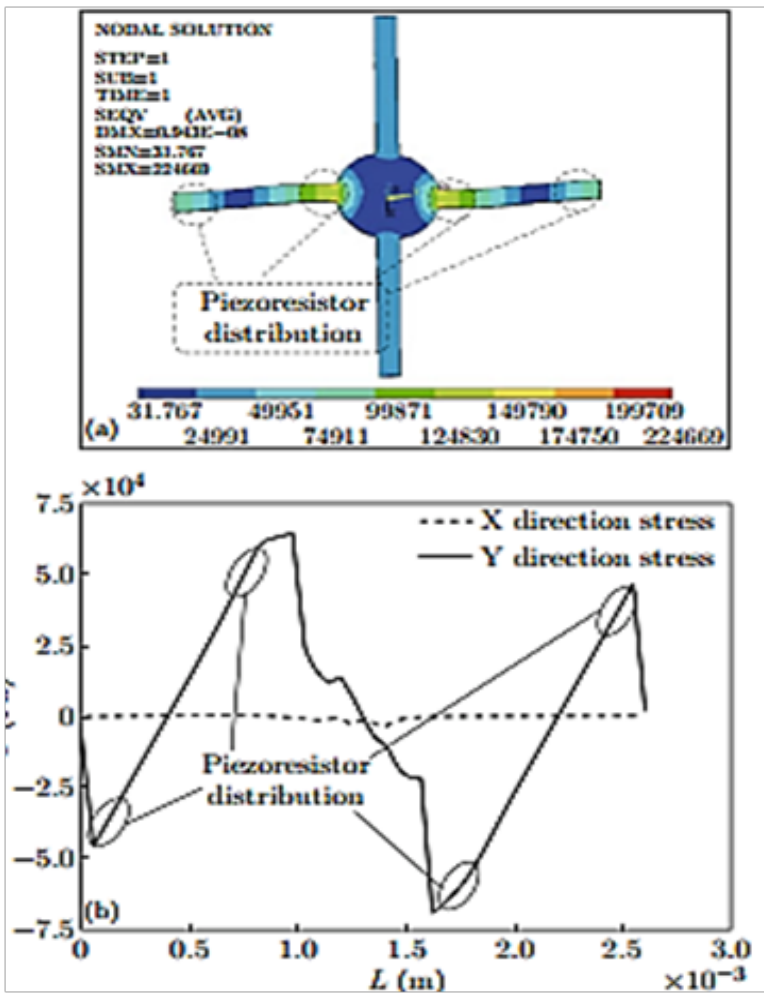

Figure 6 (A) The stress-contour of cross-beam; (B) Curve of cross-beam stress distribution.

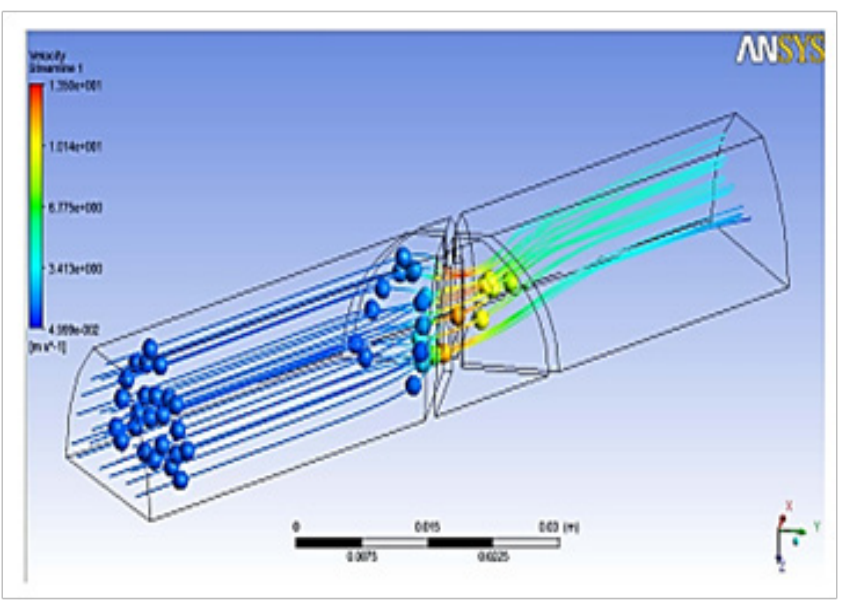

Figure 7 The cohesive energy model of "umbrella-type" packaged structure.

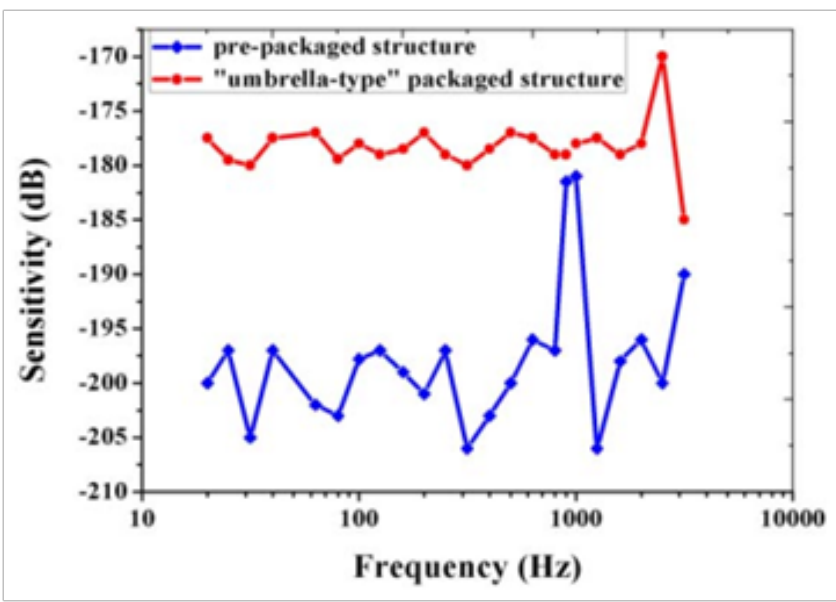

Figure 8 Frequency response of pre-packaged and "umbrella-type" packaged MEMS Bionic Vector Hydrophone.

Based on the 2DVH, the piezo resistive strips of the $z$-axis are arranged on the $y$-axis according to the stress distribution on the beam. In addition, a 'fitness-wheel' structure is adopted to remove the nonlinear output problem, further increase the sensitivity of the $z$-axis and promise the uniform tri-axial output. Research requirements are driving the increase of the detection dimensions of MEMS hydrophones from $2 \mathrm{D}$ to $3 \mathrm{D}$.

\section{Conclusion}

According to the test results, the hydrophone has a good frequency response in the range of $25 \mathrm{~Hz} \square 1500 \mathrm{~Hz}$, and its sensitivity can reach up to $-180 \mathrm{~dB}$. Directivity tests displayed that the hydrophone has a good "8"-shaped directivity, whose resolution was not less than 30 $\mathrm{B}$. The improvement of the hydrophone sensitivity and expanding its available band are under ongoing. simulations, confirming the correct modeling of the hydrophone as well as its prediction capability, further verifying the feasibility of detecting underwater acoustic signals by the "umbrellatype" packaged hydrophone, which establish foundation for further engineering application. As expected, this hydrophone is more miniature compared with the traditional hydrophone by means of the ingenious bionic structure and MEMS technology, and the low- 
frequency characteristic of the hydrophone is desirable because of the application of piezo resistive effect. It possesses good directional pattern in the form of " 8 " shape, and the receiving sensitivity of the hydrophone is up to $197.7 \mathrm{~dB}\left(0 \mathrm{~dB}^{1 / 4} 1 \mathrm{~V} / \mathrm{mPa}\right)$.

\section{Acknowledgments}

None.

\section{Conflict of interests}

The authors declare that there is no conflict of interests regarding the publication of this paper.

\section{References}

I. Xue C, Chen S, Zhang W, et al. Design, fabrication, and preliminary characterization of a novel MEMS bionic vector hydrophone. Microelectron J. 2007;38(II):102I-1026.

2. Leslie CB, Kendall JM, Jones JL, et al. Hydrophone for measuring particle velocity. J Acoust Soc Am. 1956;28(4):7II-7I5.

3. Bastyr KJ, Lauchle GC, McConnell JA, et al. Development of a velocity gradient underwater acoustic intensity sensor. I Acoust Soc Am. 1999;106(6):3178-3188.
4. Zhifang Fan, Jack Chen, David Bullen JZ, et al. Design and fabrication of artificial lateral line flow sensors. J Micromech Microeng. 2002; I2(5):655661 .

5. Izadi N, de Boer MJ, Berenschot JW, et al. Fabrication of dense flow sensor arrays on flexible membranes. Transducers. 2009; I075-1078.

6. Shipps JC, Deng K.A miniature vector sensor for line array applications, Proceedings of the IEEE Oceans. 2003;5:2367-2370.

7. Shipps JC,Abraham BM. The use of vector sensors for underwater port and waterway security. Proceedings of the ISA/IEEE Sensors for Industry Conference. 2004;4I-44.

8. Boston University. A Novel Micro Electro2Mechanical System (MEMS), Design for an Underwater Acoustic Field Sensor, ASA/EAA/DAGA'99. Meeting Lay Language Papers: Berlin; 1999.

9. Chan HLW, Lau ST, Kwok KW, et al. Nanocomposite ultrasonic hydrophones. Sensors Actuators A. 1999;75(3):252-256.

10. Heerfordt A, Møhl B, Wahlberg M, et al. A wideband connection to sperm whales: A fiber-optic, deep-sea hydrophone array. Deep-Sea Res. 2007;54(3):428-436.

II. Bagnoli PE, Beverini N, Falciai R, et al. Development of an erbium-doped fibre laser as a deepsea hydrophone.J Opt A:Pure Appl. 2006;8(7):535-539. 